\title{
Local Wastewater Treatment by Effective Coagulants Based on Wastes
}

\author{
Svitlana Kyrii ${ }^{*}$, Tetiana Dontsova', Iryna Kosogina', Ihor Astrelin', \\ Nataliya Klymenko' ${ }^{1}$, Daria Nechyporuk ${ }^{1}$
}

${ }^{1}$ National Technical University of Ukraine "Igor Sikorsky Kyiv Polytechnic Institute", Chemical Technology Faculty, Department of Inorganic Substances, Water Purification and General Chemical Technology, Peremohy 37, 03056 Kyiv, Ukraine

* Corresponding author’s e-mail: kysvit@gmail.com

\begin{abstract}
The purpose of the research was to synthesize new effective and cheap coagulants from industrial wastes (red mud) and study their coagulation properties on model water of textile industries, as well as develop a closed scheme of local wastewater treatment using the synthesized coagulants. Liquid coagulants, synthesized with the simple method, have better performance than commercial $\mathrm{FeCl}_{3}$, since not only iron but also aluminum and titanium compounds are present in the synthesized liquid coagulants, which makes them more effective. For the synthesized coagulants with a dose of $10 \mathrm{mg} / \mathrm{l}$, the removal efficiency of the dyes mixture from the water was $99.7 \%$, while for the commercial $\mathrm{FeCl}_{3}$, with a 2.5 times higher dose - only $95.4 \%$. In the case of creating closed scheme of treatment and usage of the treated water with the coagulation method, an additional sorption purification step from the trace concentrations of pollutants was proposed. The usage of the new coagulants, synthesized from the wastes of alumina production in the proposed scheme will not only help reduce the environmental burden by minimizing the wastewater discharges into sewerage, but also recycle the accumulated environmentally hazardous "red mud" waste into commercial products.
\end{abstract}

Keywords: red mud, waste utilization, effective coagulants, coagulation, local wastewater treatment, adsorption.

\section{INTRODUCTION}

In recent years, despite the improvement of the existing technologies in the chemical industry, the man-made environmental pollution has increased, which is reflected in greater wastes and wastewater volume. The textile industry is no exception, and the amount of wastewater polluted by contaminants of different nature (dyes, surfactants, salts) is constantly increasing. Thus, when using 1 ton of dyes, more than 225 tons of wastewater with high color and salt content is produced [Natarajan et al. 2018; Ying Ling et al. 2017; Angelova et al. 2016]. Discharging such waters to reservoirs leads to the deterioration of sanitary standards of water, as such water can be toxic to aquatic organisms due to the content of chlorides and decay products of dyes.
The wastewater containing dyes is very difficult to treat, since the dyes are stable in light and are usually resistant to aerobic digestion. The color removal is related to the destruction of the conjugated unsaturated bonds in the dye molecules. Therefore, to achieve a satisfactory treatment degree of such wastewater, it is necessary to carry out a complex of technological methods such as coagulation, filtration, adsorption on activated carbon, and the like. However, given the scarcity of water resources, it is advisable to develop the purification schemes with a high degree of pollutants extraction, which can ensure wastewater reusing [Wang Li et al. 2018].

Among the many methods of water treatment today in practice (both at municipal treatment plants and in industrial water treatment processes), the method of coagulation, which allows 
removing contaminants of different origins, is used most often. This method is simple, allows the usage of different coagulants, and is quite effective [Nabibidhendi et al. 2007, Makarchuk et al. 2017, Monastyrov et al. 2019]. However, by coagulation, it is often not possible to carry out advanced purification of water that will be reused in the production cycle, which necessitates an additional refinement operation, such as adsorption. As it is known, adsorption is one of the most common methods for the pollutants removal from wastewater since the adsorption process will produce a high-quality treated effluent and have proper design. Consequently, the traditional wastewater treatment in the textile industries is usually realized by combining the use of ironcontaining coagulants with secondary treatment by sorption on activated carbon. However, the high cost of activated carbon and the complexity of its regeneration necessitate finding the ways of its modification to develop performance or reduce its costs by improving pre-treatment. Therefore, it is important to develop new high-efficiency and cheap coagulants. For such coagulants, cost reductions are possible, for example, by substituting raw materials with the waste from various industries containing iron and aluminum compounds. Such wastes in large quantities are waste from alumina production known as "red mud". The issue of the accumulated waste utilization in large quantities becomes more and more acute every year. The circular economy technologies or resource-saving technologies that could reduce the accumulated amount of industrial waste and make the process of obtaining the finished product cheaper are increasingly urgent [Mazari et al. 2018]. It is possible to implement effective circular economy technology, for example, by using the red mud waste generated from the alumina production. The total amount of red mud produced every year in the world fluctuates from 60 to 120 million tons. The accumulation of red mud is a problem not only in Ukraine but also in such countries as the USA, China, Turkey, Hungary, India, Russia, Canada. Therefore, the problem of rational use and recycling of red mud deserves great attention. The problem of red mud storage leads to the pollution of soil, surface water, and the air due to wind deflation, which necessitates its disposal for environmental disaster prevention, such as the one that occurred in Hungary in 2010.

It has been established that red mud can be used in various industries, for example, in ferrous metallurgy, for the production of pig iron, alumina, and various separate components, the building industry for making bricks and cement, ceramic, glass [Dong-Yan et al. 2012; Nan et al. 2010; Kalkan, 2006]. Due to the high content of iron oxides (up to 55\%) and aluminum (up to $30 \%$ ), red mud can be used in the water purification technology as coagulants or adsorbents after their synthesis processes [Kulishenko et al. 2018; Kyrii et al. 2018]. Therefore, the synthesis of new efficient coagulants from the alumina waste is a very relevant and promising area both for waste utilization and the creation of effective wastewater treatment schemes for the textile industries with coagulation usage. At the same time, the creation of local schemes of water treatment using the coagulants obtained from the waste of alumina production will solve several problems at once:

- Reduction of the ecological load on the environment through the creation of a closed scheme of local wastewater treatment;

- Development of new effective coagulants of complex action from secondary raw materials, namely from the wastes of alumina production;

- Utilization of a large amount of environmentally hazardous wastes.

This complex approach will allow developing a local closed scheme for advanced wastewater treatment from dyes of different origins.

The purpose of the work is a synthesis of new effective and cheap coagulants from industrial wastes (red mud) and studying its coagulation properties on model waters of textile industries, as well as development of a closed scheme of local wastewater treatment using synthesized coagulants.

\section{MATERIAL AND METHODS}

\section{Dyes characteristics}

Aqueous solutions of cationic (methylene blue) and anionic (Congo red) dyes were used to test the coagulants. The characteristics of the dyes are presented in Table 1.

Using the Hyperchem program, the $3 \mathrm{~d}$ models of the dye molecules were constructed, and their geometric optimization was carried out (Fig. 1). The characteristic size of the molecule (D in Table 1) was taken to be its largest linear size [Kyrii et al. 2018]. 
Table 1. Dye characteristics

\begin{tabular}{|c|c|c|c|c|}
\hline Dye & The dye nature & $\mathrm{Dm}$ \\
\hline Methylene blue & Structural formula & cationic \\
\hline Congo red
\end{tabular}

Model solutions of methylene blue $(\lambda=670 \mathrm{~nm})$ with concentration $10 \mathrm{mg} / \mathrm{l}$, Congo red $(\lambda=540 \mathrm{~nm})$ with concentration $10 \mathrm{mg} / 1$ and their mixture $(\lambda=664 \mathrm{~nm})$ with concentration of each $10 \mathrm{mg} / \mathrm{l}$ were used.

\section{Characteristics of red mud}

For the coagulant synthesis, the red mud of the Mykolaiv alumina plant (Ukraine) was used. The chemical composition of red mud was investigated by means of X-ray diffraction analysis, the results of which are shown in table 2.

\section{Coagulant synthesis}

The coagulant synthesis was carried out by treating the original red mud with $15 \%$ acid in a 1: 1 mass ratio and boiled at $100^{\circ} \mathrm{C}$ for 1 hour. The resulting suspension was filtered, and the filtrate was used as a liquid coagulant. When using $\mathrm{HCl}$, the $\mathrm{LC}_{\mathrm{Cl}}$ coagulant was received, whereas with $\mathrm{H}_{2} \mathrm{SO}_{4}-\mathrm{LC}_{\mathrm{SO} 4}$ was obtained. The elemental composition of the synthesized coagulants by major components was determined by the X-ray fluorescence method on an Oxford XSupreme instrument.

\section{Jar-test}

A conventional jar test apparatus was used in the experiments for the coagulation of textile wastewater by different coagulants: standard coagulant $\mathrm{FeCl}_{3}$, liquid coagulant synthesized with the addition of hydrochloric acid $\left(\mathrm{LC}_{\mathrm{CI}}\right)$ and a liquid coagulant with the addition of sulfate acid $\left(\mathrm{LC}_{\mathrm{SO} 4}\right)$. The $\mathrm{pH}$ was controlled by adding a strong base $(\mathrm{NaOH})$ to 7 . After the desired amount of coagulants were added to the wastewater model, the beakers were agitated at various mixing time and speed, which consist of rapid mixing $(250 \mathrm{rpm})$ for 2-3 minutes and slow mixing $(30 \mathrm{rpm})$ for 5 minutes. The samples were measured for color removing comparing with the initial concentration. After the agitation was stopped, the suspension was allowed to settle for 10-20 minutes. Finally, the sample was collected using a pipette from the top inch of supernatant for color measurements, which representing the final concentration. All tests were performed at an ambient temperature of $20^{\circ} \mathrm{C}$. a)

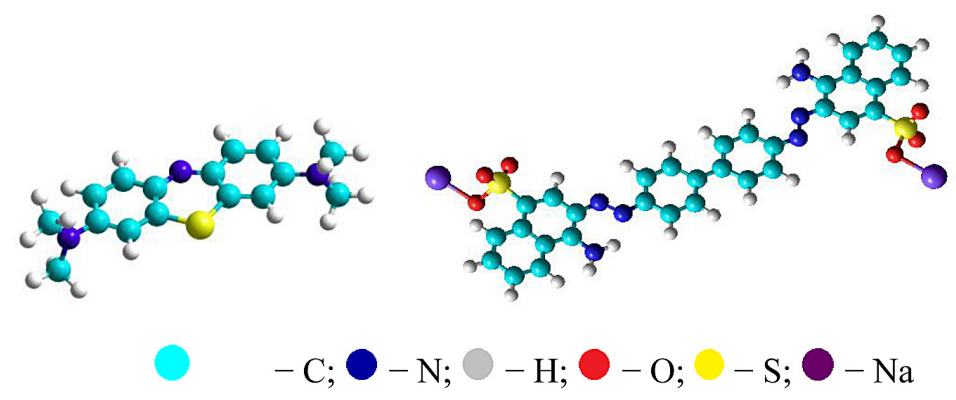

Fig. 1. 3D models of dye molecules; $a$ - Methylene blue; $b$ - Congo red 
Table 2. Chemical composition of red mud

\begin{tabular}{|c|c|}
\hline Component & $\begin{array}{c}\text { Content of components } \\
\text { in red mud,\% wt. }\end{array}$ \\
\hline $\mathrm{Fe}_{2} \mathrm{O}_{3}$ & $40-55$ \\
\hline $\mathrm{Al}_{2} \mathrm{O}_{3}$ & $14-18$ \\
\hline $\mathrm{SiO}_{2}$ & $5-10$ \\
\hline $\mathrm{CaO}$ & $5-10$ \\
\hline $\mathrm{TiO}_{2}$ & $4-6$ \\
\hline $\mathrm{Na}_{2} \mathrm{O}$ & $2-4$ \\
\hline
\end{tabular}

\section{Adsorption procedure}

The analysis of the sorption properties was carried out by constructing the adsorption isotherms of dyes (removal of methylene blue and Congo red). For the sorption studies, a series of $250 \mathrm{~mL}$ glass flasks were filled with $100 \mathrm{~mL}$ of model wastewater solution of different initial contaminant concentrations $\left(1-10 \mathrm{mg} \cdot \mathrm{L}^{-1}\right)$. A fixed amount of sorbent material $(0.1 \mathrm{~g})$ was added into each flask and stirred (350 rpm) for 5 hours at thermostatic water bath shaker $\left(20 \pm 2{ }^{\circ} \mathrm{C}\right)$ to ensure complete sorption equilibrium. After adsorption, spent sorption materials were separated by centrifugal separation. The equilibrium concentrations of dyes in the solutions after adsorption treatment were determined by means of the photometric method using UV-5800PC Spectrophotometer.

\section{RESULTS AND DISCUSSIONS}

\section{Characterization of coagulants}

The resulting by acid-thermal activation comprehensive liquid coagulants were characterized by the following chemical composition (by main components): coagulant $\mathrm{LC}_{\mathrm{Cl}} \mathrm{Fe}-82.87 \%$, $\mathrm{Al}-5.12 \%$, $\mathrm{Ti}-0.98 \%, \mathrm{Ca}-1.78 \%$; and coagulant $\mathrm{LC}_{\mathrm{SO} 4} \mathrm{Fe}-83.24 \%, \mathrm{Al}-1.95 \%$; $\mathrm{Ti}-1.44 \%$.

\section{Coagulation}

The efficiency of using synthesized liquid coagulants was tested on model waters containing cationic dyes (methylene blue), anionic dyes (Congo red), and mixtures thereof. The results obtained for the synthesized coagulants were compared with those for the commercial coagulant $\mathrm{FeCl}_{3}$ ("NeoSynthesis", Ukraine).

The coagulation was examined using a $\mathrm{pH}-\mathrm{ad}-$ justed jar test. For the synthesized coagulants, the
$\mathrm{pH}$ was adjusted to 7 , for the commercial coagulant $\mathrm{FeCl}_{3}$ - to 9 (at the beginning of flocs formation). Moreover, commercial coagulant $\mathrm{FeCl}_{3}$ experienced difficulties in undergoing coagulation, namely the slow formation and coarsening of micro-flocs with subsequent long deposition. In turn, the usage of the synthesized coagulants $\mathrm{LC}_{\mathrm{Cl}}$ and $\mathrm{LC}_{\mathrm{SO} 4}$ contributed to the high speed of formation, adhesion and sedimentation of flocs in the treatment of model wastewater contaminated with dyes of different origins.

As a result of research, the optimal conditions for carrying out the coagulation process for different types of coagulants were determined. It was established that for the treatment of water contaminated with one type of dye, the optimal doses are the same for the three coagulants and equal to $10 \mathrm{mg} / \mathrm{l}$. For the treatment of the model wastewater polluted with a mixture of dyes, the optimal dose of commercial coagulant $\mathrm{FeCl}_{3}$ is 2.5 times higher $(25 \mathrm{mg} / \mathrm{l})$ than for the synthesized coagulants $(11 \mathrm{mg} / \mathrm{l})$. In this case, the removal efficiency of dyes ranges from $94 \%$ to $99.7 \%$, depending on the type of dye and coagulant (Fig. 2).

Therefore, according to the experimental data, it was found that with the presence of cationic dyes (methylene blue) in water, coagulant $\mathrm{LC}_{\mathrm{SO} 4}$ is the most effective (almost $98 \%$ removal degree). Perhaps, this is due to a stronger bond between the counterions, which are part of the double electric layer, and the ions of cationic type of pollutant, which are strongly held by electrostatic and adsorption forces near the surface of the coagulant during chemical bonding. In the presence of anionic dyes in water (Congo red), the $\mathrm{LC}_{\mathrm{Cl}}$ coagulant is the most effective (the degree of dye removal is almost $99 \%$, while for commercial $\mathrm{FeCl}_{3}$ coagulant - only $96.5 \%$ ). The difference in the efficiency between the commercial $\mathrm{FeCl}_{3}$ and the synthesized $\mathrm{LC}_{\mathrm{Cl}}$ is explained by its complex action, since the $\mathrm{LC}_{\mathrm{C}}$, in addition to the iron compounds, also has aluminum and titanium compounds.

In the presence of several types of pollutants of different nature in the water (dyes mixture), there is an increase in the coagulation efficiency to $95.4 \%$ for commercial $\mathrm{FeCl}_{3}$, and to $98.3 \%$ and $99.7 \%$ for $\mathrm{LC}_{\mathrm{SO} 4}$ and $\mathrm{LC}_{\mathrm{Cl}}$, respectively, due to the synergistic effect between dyes and the iron, aluminum and titanium compounds in coagulants. That is, the presence of different types of contamination in water will facilitate a more efficient and intensive coagulation process. 


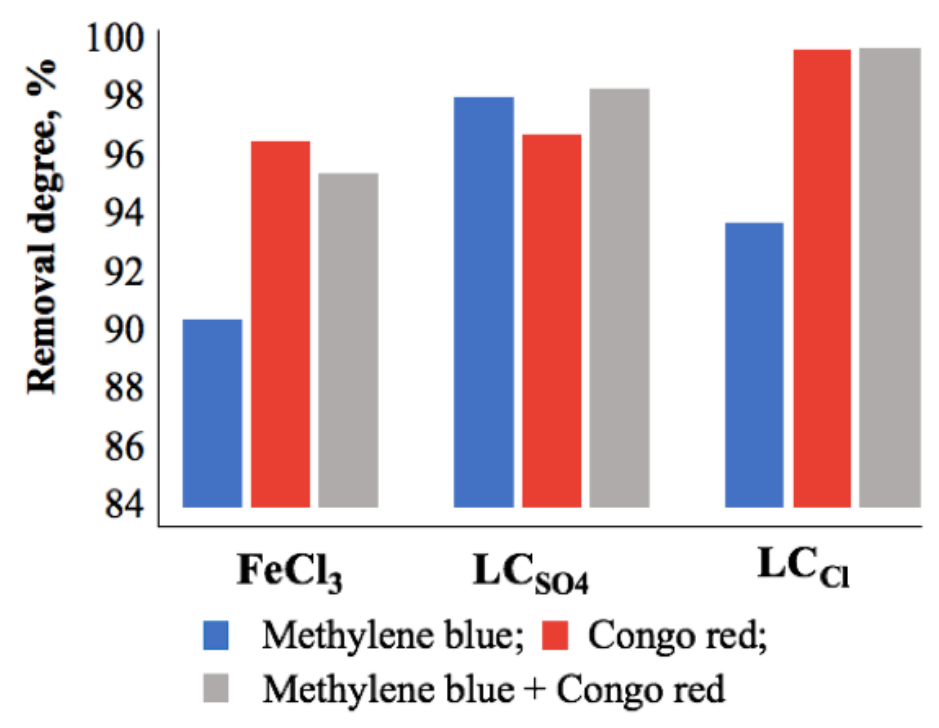

Fig. 2. Comparison of the effectiveness of different coagulants using for various pollutants at the optimal doses

In addition to the dose of coagulants, the rate of coagulation process passage, namely the rate of formation, adhesion, and deposition of microflocs, was investigated. Thus, in the water treatment under the same coagulation conditions for all three coagulants, it was found that the time of micro-flocs formation and their coarsening did not exceed 2 min for $\mathrm{LC}_{\mathrm{Cl}}, 2.5$ min for $\mathrm{LC}_{\mathrm{SO} 4}$ and $3.6 \mathrm{~min}$ for $\mathrm{FeCl}_{3}$. This indicates that the rate of coarsening and sedimentation depends on coagulant nature. Figure 3 shows the photos of the water system state after 5 minutes of coagulation during the micro-flocs deposition stage, under the same process conditions for three types of coagulants, illustrating the above.

Thus, when purifying the water contaminated with a mixture of dyes (methylene blue and Congo red) by synthesized coagulants, there is an accelerated process of micro-flocs aggregation - by 3 times for $\mathrm{LC}_{\mathrm{Cl}}$ and 1.5 times for $\mathrm{LC}_{\mathrm{SO} 4}$, respectively, in comparison with the commercial $\mathrm{FeCl}_{3}$ coagulant. The duration of flocs sedimentation does not exceed 30 minutes with using synthesized complex coagulants $\mathrm{LC}_{\mathrm{Cl}}$ and $\mathrm{LC}_{\mathrm{SO} 4}$, and 90 minutes for commercial $\mathrm{FeCl}_{3}$.

Since the synthesized coagulants have a complex composition, the presence of aluminum in them contributes to the formation of a more developed flocs surface and accelerates the formation of their branched structure, while the presence of iron promotes the formation of larger flocs and their rapid deposition.

Coagulation of the model wastewater contaminated with dyes (for example Congo red) was carried out by the following reactions (for $\mathrm{Fe}$ as the main component):

$$
\begin{gathered}
{\left[\mathrm{Fe}\left(\mathrm{H}_{2} \mathrm{O}\right)_{6}\right] \mathrm{SO}_{4}+2 \mathrm{NaOH}=} \\
=\left[\mathrm{Fe}\left(\mathrm{H}_{2} \mathrm{O}\right)_{6}\right](\mathrm{OH})_{2}+\mathrm{Na}_{2} \mathrm{SO}_{4}, \\
4\left[\mathrm{Fe}\left(\mathrm{H}_{2} \mathrm{O}\right)_{6}\right](\mathrm{OH})_{2}+\mathrm{O}_{2}+2 \mathrm{H}_{2} \mathrm{O}= \\
=4\left[\mathrm{Fe}\left(\mathrm{H}_{2} \mathrm{O}\right)_{6}\right](\mathrm{OH})_{3},
\end{gathered}
$$
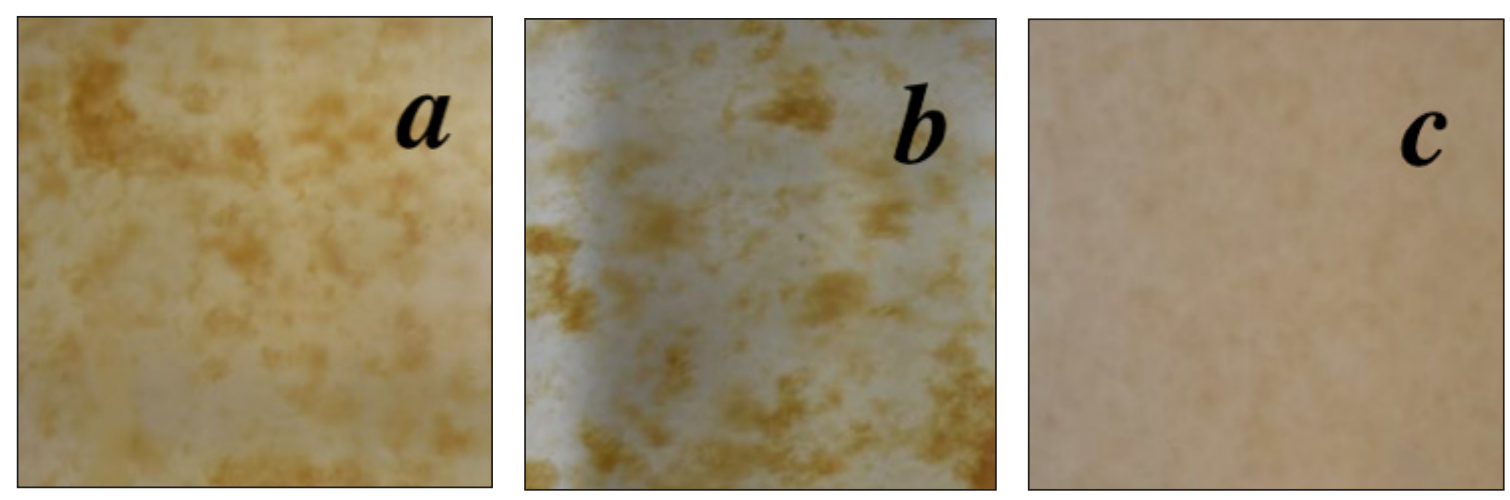

Fig. 3. Flocs formation by different coagulants (magnifications in 4 times) 
Table 3. Langmuir, Freundlich, Temkin isotherms constants for the dyes adsorption

\begin{tabular}{|c|c|c|c|c|c|c|c|c|}
\hline Model & \multicolumn{3}{|c|}{ Langmuir } & \multicolumn{3}{c|}{ Freundlich } & \multicolumn{2}{c|}{ Temkin } \\
\hline Model water & $\mathrm{R}^{2}$ & $\mathrm{~K}_{\mathrm{L}}$ & $\mathrm{a}_{\infty}, \mathrm{mg} / \mathrm{g}$ & $\mathrm{R}^{2}$ & $\mathrm{~K}_{\mathrm{F}}$ & $\mathrm{n}$ & $\mathrm{R}^{2}$ & $\mathrm{~B}, \mathrm{~J} / \mathrm{mol}$ \\
\hline Methylene blue & 0.92 & 0.14 & 7.43 & 0.89 & 2.97 & 1.81 & 0.89 & 2.17 \\
\hline Congo red & 0.92 & 0.02 & 50.51 & 0.94 & 1.96 & 0.62 & 0.95 & 5.35 \\
\hline
\end{tabular}

The dye sorption is due to the exchange reactions of the $\mathrm{OH}$ group on the acid anion in the outer sphere of the aqua-complex:

$$
\begin{aligned}
& {\left[\mathrm{Fe}\left(\mathrm{H}_{2} \mathrm{O}\right)_{6}\right](\mathrm{OH})_{3}+\mathrm{RSO}_{3} \mathrm{Na}=} \\
& =\left[\mathrm{Fe}\left(\mathrm{H}_{2} \mathrm{O}\right)_{6}\right](\mathrm{OH})_{2}\left(\mathrm{RSO}_{3}\right)+\mathrm{NaOH} \text {, }
\end{aligned}
$$

where: $R-\mathrm{C}_{32} \mathrm{H}_{22} \mathrm{~N}_{6} \mathrm{NaO}_{3} \mathrm{~S}$ for the Congo red dye.

Therefore, it can be concluded that for water reusing, it is sufficient to use the coagulation stage, but using liquid effective complex coagulants, which allow purifying the water up to $99.7 \%$ in a short period. However, in the case of using closed wastewater treatment schemes, given the repeated use of purified water, a partial accumulation of residual concentrations of pollutants is possible, which necessitates an advanced water purification step, such as sorption.

\section{Adsorption study}

In multiple uses of purified water in closed cycles when there is a necessity of advanced tertiary treatment (for example, when creating a closed circuit of water without the formation of sewage), the finishing of water purification by means of the adsorption method on activated carbon is proposed. Therefore, to develop a universal scheme, it is necessary to further study the adsorption of organic pollutants on activated carbon. For this purpose, an adsorption study was conducted for the model water containing cationic dye (methylene blue) and anionic dye (Congo red), with a concentration of $10 \mathrm{mg} / \mathrm{l} \mathrm{each}$. The obtained data from the sorption isotherm were processed using the mathematical models of Langmuir, Freundlich, and Temkin (Table 3).

As can be seen from Table 3, the limiting sorption of the Congo red dye, calculated according to the Langmuir model, is $50.51 \mathrm{mg} / \mathrm{g}$, which is 6.8 more than for methylene blue. It may be due to the higher binding energy between the surface of activated carbon and the dye Congo red, which is confirmed by the calculated Temkin B constant. The high determination coefficient of the Langmuir model indicates the monolayer filling of the pollutant in the sorbent layer during the sorption process.

The physical nature of the sorption was confirmed by the IR-spectroscopy of three samples: initial activated carbon F300, methylene blue dye, and saturated activated carbon F300 with methylene blue dye (Fig. 4). The absence of displacement of the major peaks in the range $900-$ $1400 \mathrm{~cm}^{-1}$ for -COO-; $1200-1450 \mathrm{~cm}^{-1}$ for $\mathrm{NH}^{+}$; $1900-2000 \mathrm{~cm}^{-1}$ for $-\mathrm{C}(\mathrm{O}) \mathrm{O}-; 2300-2350 \mathrm{~cm}^{-1}$ for $-\mathrm{C} \equiv \mathrm{N} ; 2850-3050 \mathrm{~cm}^{-1}$ for $-\mathrm{CH}-;-\mathrm{CH}_{2}-$ indicates that the pollutants are not destroyed and adsorbed in the pores of the carbon. It is related, probably, with the interaction of the surface functional groups of the activated carbon with the molecules of the pollutants.

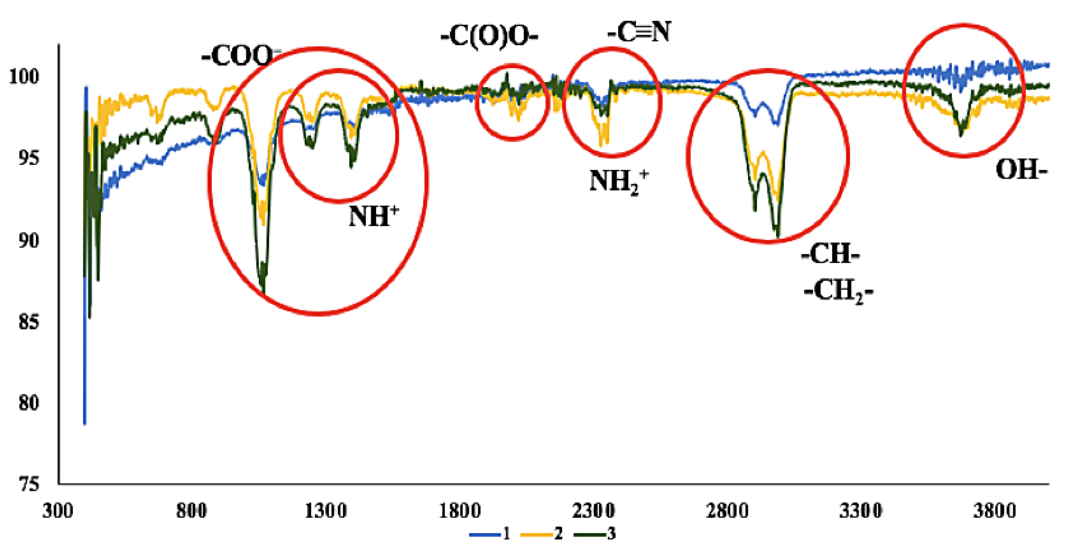

Fig. 4 IR spectrum of AC A300 (1), Methylene blue (2), AC F300 + Methylene blue (3) 


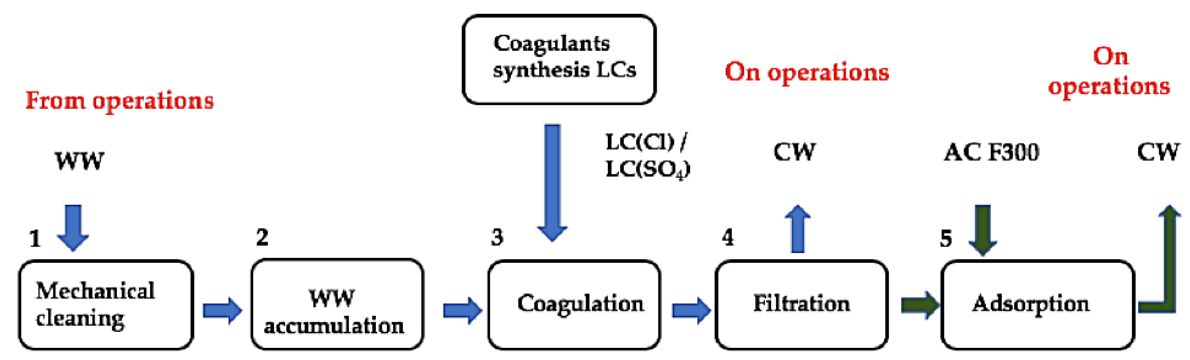

Fig. 5 Conceptual scheme of local wastewater treatment using effective coagulants based on waste: WW - wastewater; CW - cleaned water; RM - red mud; LC - liquid coagulant; AC F300 - activated carbon Filtrasorb 300

\section{Conceptual scheme of wastewater treatment for closed cycles}

Figure 5 shows the developed local purification scheme, based on the conducted research, which includes the coagulation and adsorption step.

According to the proposed conceptual scheme, the process proceeds in 5 stages. The synthesis of coagulants occurs by acid-thermal activation of red mud with mineral acids. The result receives complex liquid $\mathrm{LC}_{\mathrm{Cl}}$ and $\mathrm{LC}_{\mathrm{SO} 4}$ coagulants containing the iron, aluminum, and titanium compounds. The finished product can be stored and used on any treatment plant. It is necessary to remove suspended particles from wastewater (stage 1) and averaged their composition (stage 2) to achieve high efficiency of treatment by coagulation. The foremost treatment step is coagulation (stage 3 ), then the filtration step on the microfiltration membranes (stage 4), which contributes to the separation of impurities in the event of a violation of the technological regime. Afterwards, the water can be reused for technical operations. In the case of the accumulation of residual concentrations of contaminants, it is suggested to conduct advanced secondary treatment of water by adsorption (step 5).

\section{CONCLUSIONS}

The scheme of local wastewater treatment with the use of new effective complex-action coagulants synthesized from industrial waste by means of a simple method has been developed. The synthesis of such coagulants was carried out by acid-thermal treatment of waste of alumina production red mud. The created liquid complex action $\mathrm{LC}_{\mathrm{Cl}}$ and $\mathrm{LC}_{\mathrm{SO} 4}$ coagulants showed high efficiency (up to $99.7 \%$ ) while removing dyes of different nature.

It was found that in the case of a mixture of anionic and cationic dyes removal from aqueous solutions, the dose of synthesized coagulants is half of that corresponding to the commercial $\mathrm{FeCl}_{3}$ coagulant. The duration of coagulation by synthesized coagulants is reduced by $1.5-3$ times compared to the traditional $\mathrm{FeCl}_{3}$ coagulant due to the accelerated processes of formation, agglomeration, and sedimentation of the formed particles. It was shown that liquid complex coagulant $\mathrm{LC}_{\mathrm{SO} 4}$ is the most effective due to its more stable operation and independence from the type and amount of water contaminants.

The patterns of adsorption purification from trace concentrations of dyes on activated carbon have been investigated. Their mathematical processing and analysis of the IR-spectra of the initial and contaminated samples of the sorption material, as well as the methylene blue dye, showed the physical nature of the interaction of the dyes with activated carbon.

On the basis of the identified patterns, a closed scheme of local wastewater treatment of the textile industry has been developed, which will minimize the amount of sewage discharged into sewage and reservoirs due to the reuse of water. The scheme provides for the treatment of wastewater contaminated with dyes by the coagulation method, to return it to the technological cycle using highly effective liquid coagulants of complex action. When advanced secondary treatment of water from trace concentrations of contaminants after multiuse is appropriate, it is planned to purify water with adsorption on activated carbon. 


\section{REFERENCES}

1. Angelova R. et al. 2016. Magnetically modified Sargassum horneri biomass as an adsorbent for organic dye removal. Jour. Clean. Prod. 137, 189-194. https://doi.org/10.1016/j.jclepro.2016.07.068

2. Dong-Yan L., Chuan-Sheng W. 2012. Stockpiling and Comprehensive Utilization of Red Mud Research Progress. Materials. 5, 1232-1246. https:// doi.org/10.1016/j.jes.2017.03.011

3. Kalkan, E. 2006. Utilization of red mud as a stabilization material for the preparation of clay liners. Eng. Geol. 87, 220-229.

4. Kulishenko A.E. et al. 2018. The Use of Products of Recycling Waste of Aluminum Manufacturing as a Coagulant when Purifying Highly Colored Natural Water. J. Water Chem. Technol. 40 (5), 265-271. doi.org/10.3103/S1063455X1805003X

5. Kyrii S.O. et al. 2018. Investigation of the properties of activated carbon modified by wastes of alumina production. Vopr. Khim. Khimich. Tekhnol. 2, 70- 78 .

6. Kyrii S. et al. 2018. A comparison of carboncontaining and clay sorption materials and (nano) composites based on them. Norweg. Jour. Develop. Intern. Sci. 25, 19-27.

7. Mazari L. et al. 2018. Evaluating Reuse of Alum Sludge as Coagulant for Tertiary Wastewater Treatment. J. Environ. Eng. 144(12): 04018119.
8. Monastyrov M. et al. 2019. Electroerosion dispersion, sorption and coagulaton for complex water purification: electroerosion waste recycling and manufacturing of metals, oxides and alloys nanopowders. Nanotech. Percep. 15, 48-57.

9. Nabibidhendi G.R. et al. 2007. Evaluation of industrial dyeing wastewater treatment with coagulants and polyelectrolyte as a coagulant aid. Iran. J. Environ. Health. Sci. Eng. 4 (1), 29-36.

10. Nan X.L. et al. 2010. Comprehensive utilization and analysis of Chinese red mud. Process. Eng. 10, 264-270.

11. Natarajan S. et al. 2018. Recent advances based on the synergetic effect of adsorption for removal of dyes from waste water using photocatalytic process. J. Environ. Sci. 65, 201-222.

12. Makarchuk Oksana et al. 2017. Magnetic Mineral Nanocomposite Sorbents for Wastewater Treatment. Jour. Nanomater. 2017, 1-7. https://doi. org/10.1155/2017/8579598

13. Wang Li et al. 2018. Microwave assisted modification of activated carbons by organic acid ammoniums activation for enhanced adsorption of acid red 18. Powd. Techn. 323, 230-237. https://doi. org/10.1016/j.powtec.2017.10.021

14. Ying Ling Y. et al. 2017. Extraction of malachite green from wastewater by using polymer inclusion membrane. Jour. Envir. Chem. Eng. 5(1), 785-794. https://doi.org/10.1016/j.jece.2017.01.001 